\title{
THE HARWELL NEUTRON PROJECT
}

\author{
By Dr. M. J. POOLE and Dr. E. R. RAE
}

Atomic Energy Research Establishment, Harwell, Didcot, Berks

\begin{abstract}
A
LABORATORY has been set up at the Atomic

Energy Research Establishment, Harwoll'1, to exploit the possibility of using a linear electron accelerator as a pulsed noutron-source for time-offlight studies. These studies increase our knowledge of highly excited states of compound nuclei.

The first machine to be used in this way produced electrons of energy $3 \mathrm{MeV}$., and was superseded in 1952 by a $15-\mathrm{MeV}$. machine, which produced photodisintegration and photo-fission neutrons from a block of uranium which served as target for the electrons. In that accelerator the duration of the electron pulses could be made as short as $0.1 \mu \mathrm{sec}$. by pulsing the electron gun, and the beam current during a pulse was of the order of $25 \mathrm{~m}$.amp. at $13.5 \mathrm{MeV}$. under full-load conditions (400 pulses/sec.). In these circumstances the rate of production of neutrons during the pulse was $10^{14}-10^{15}$ neutrons/sec.
\end{abstract}

The new laboratory has been designed around a much more powerful machine than either of its forerunners, since it is capable of providing a beam current during the pulse of the order of 400 m.amp. at $30 \mathrm{MeV}$. Using this in conjunction with a natural uranium target, a rate of emission of $2 \times 10^{16}$ neutrons/see. is possible. However, a further increase in the available neutron output has been obtained by building a special target (called the neutron booster) which is a multiplying sub-critical uranium-235 assembly, and by this means a ten-fold increase in the neutron output has been obtained. Thus the final rate of production of neutrons during the pulse is $3 \times 10^{17}$ neutrons/sec. (A factor of 1.5 is accounted for by the difference in the production-rate of primary neutrons in natural uranium and uranium-235.)

In the time-of-flight experiments the neutrons are partly moderated in flat water tanks, $2 \mathrm{~cm}$. thick, placed on three sides of the neutron booster. A layer of boron-10 metal placed between these tanks and the uranium-235 core prevents return of slow neutrons to the uranium-235 and so avoids serious distortion of the pulse shape. Some nine windows lead through the shielding and allow neutrons to pass down evacuated 'flight tubes' arranged radially about the neutron source and so reach the experimental apparatus. In this way the time-of-flight, and so the energy of the noutrons associated with some nuclear process, can be measured by recording the time-interval between the accelerator pulse and the time of arrival of the neutron. The recording of the times of arrival of the neutrons is carried out by multi-channel time analysers, one of which is associated with each individual flight path. The ability to carry out a number of time-of-flight experiments simultaneously in this way is one of the main advantages of a pulsed accelerator over a fast chopper as a pulsed neutronsource. The other advantage is the relative ease with which neutron pulses as short as $0.25 \mu$ sec. are obtainable.
Experiments in progress include measurements of neutron partial cross-sections for seattering, capture and fission, in addition to total cross-sections. From these measurements, the parameters of nuclear levels extending for several tens of $\mathrm{keV}$. above the neutron binding energy are extracted. These parameters include the level-spacings, the neutron, radiation and fission widths, and the angular momenta. Another type of experiment is the study of the hard $\gamma$-rays arising from the capture of a neutron into various levels of the compound nucleus above the binding energy. An analysis of the relative strengths of these energetic $\gamma$-rays in different levels in the same compound nucleus demonstrates the random fluctuations that oceur in the strength of a particnlar $\gamma$-ray transition.

The new machine should make possible a much more thorough investigation of the statistical laws governing the distributions of the parameters of nuclear levels, and may help to clear up certain mysteries which still surround the phenomenon of nuclear fission. In addition to these studies of neutron interactions, the investigation of a variety of photo-nuclear phonomena is also planned.

In the field of reactor physics, the pulsed-source method has been extended to allow the measurement of neutron spectra in reactor lattices. A knowledge of these spectra is of great importance in calculating the detailed performance of reactors, particularly the phenomena which govern the amount of permissible burn-up. Calculation is in principle possible once a detailed knowledge of the neutron-scattering law is known; but neither experimental results nor methods of calculation are yet sufficiently developed to make this feasible, and recourse has to be made to direct experiment. In this method the target of the pulsed machine is placed inside a section of the lattice of interest, and the neutrons are moderated down to the spectrum characteristic of that lattice. This spectrum is then measured by the usual time-offlight method.

The increased intensity from the new accelerator will make possible the performance of more complex experiments in which a pulse of neutrons is introduced into a moderator and the spectrum measured by using a synchronized neutron shutter (chopper) which opens a known time after the electron pulse. In this way it will be possible to follow the change in neutron spectrum during the last stages of slowing down, and to make a direct comparison with some of the theories of neutron thermalization available. It will also make possible the direct observation of the so-called 'neutron cooling', that is, the reduction in mean energy of neutrons in a moderator due to preferential escape of fast neutrons.

${ }^{1}$ Poole, M. J., Wiblin. E. R., et al., Proc. Sccond Int. Conf. on Peaceful Uses of Atomic Energy, Geneva, 1958 (Paper P/59). 